\title{
LA ACREDITACIÓN EN LA GENERACIÓN DE INFORMACIÓN SOBRE LA CALIDAD DE LA EDUCACIÓN SUPERIOR
}

\section{Introducción}

Clark (1983) distingue tres polos de coordinación del sistema de educación superior: los intereses académicos organizados; el mercado y la sociedad, y la autoridad estatal. Cada uno de ellos se asocia a diferentes funciones e intereses. Los intereses académicos componen el núcleo corporativo de las instituciones de educación superior; el mercado y la sociedad representan el punto de encuentro entre la oferta y demanda de educación superior, dinamizando el crecimiento del sistema, y el Estado ejerce el rol de regulación, orientado a articular el funcionamiento de las instituciones de educación terciaria con la satisfacción de las demandas sociales.

El sistema de educación superior en Chile no se adecua necesariamente a un estado de equilibrio en sus intercambios recíprocos. Es más norma que excepción el hecho de que un polo del sistema adquiera preponderancia sobre los otros, generando un modo desajustado de desarrollo. La educación superior en Chile, como plantea Brunner (2008), se distingue por estar dirigida por intereses de mercado. Ello ha incentivado la apertura de las instituciones a las expectativas de la sociedad, motivando el perfeccionamiento de los instrumentos que garantizan el interés público. No obstante, el desequilibrio del sistema hacia el polo del mercado se ha asociado con problemas en la calidad de la oferta. Por ejemplo, los programas especiales de baja calidad y dudosa pertinencia, junto con las sedes de instituciones de educación superior en precarias condiciones de operación, reflejan el funcionamiento desajustado del sistema. En consecuencia, la concepción del sistema de educación superior como integración entre sus partes es más bien el resultado de un esfuerzo de regulación que una condición dada de antemano. 
La información sobre la educación superior en Chile puede ser interpretada en virtud del enfoque planteado. De acuerdo con el interés predominante en cada subsistema, se produce información específica. Las instituciones generan información para su gestión interna y para favorecer su posicionamiento externo. El mercado y la sociedad, por su parte, demandan y producen información acerca de la oferta académica. Por último, el Estado genera información estadística para caracterizar el funcionamiento del sistema y asignar los recursos.

La composición de la información a partir de fuentes diversas no asegura un relato consistente sobre la calidad de la educación terciara. Por ejemplo, en el caso de predominar el interés del mercado, las instituciones se verán presionadas a darse a conocer principalmente a través de la publicidad. Por otro lado, cuando el sistema está determinado por los intereses académicos, probablemente no sean suficientes los incentivos para informar al mercado acerca de las características de la oferta académica.

Un sistema de educación superior de carácter masivo, diverso y heterogéneo en cuanto a la calidad y prestigio de instituciones y programas, como el chileno, constituye un sistema exigente en cuanto a demandas de información (Brunner, 2004). Así, de acuerdo con el reciente informe de la Organisation for Economic Co-operation and Development (OECD) (2009), uno de los principales problemas del sistema de educación superior en Chile es que carece de información precisa y de calidad necesaria para una evaluación acuciosa de su desempeño y de sus áreas prioritarias, al mismo tiempo que falta información detallada y confiable para el diseño de políticas.

Por esta razón, para el polo regulatorio se vuelve un desafío crucial incentivar la generación de información que diferencie entre los niveles de calidad existentes en la educación superior. Este debate fue justamente el que acompañó el proceso legislativo de la Ley 20.129, que crea a la Comisión Nacional de Acreditación (CNA). La pregunta era sobre la necesidad de generar una instancia de regulación para asegurar la calidad, si -de acuerdo a algunas posiciones- lo que hacía falta era proporcionar información que transparentara las 
características de oferta, con el fin de apoyar la toma de decisiones por parte de la demanda.

Nuestra perspectiva es que la acreditación constituye un mecanismo de regulación que se introduce al interior de la gestión de las instituciones de educación superior, combinándose con los intereses académicos y generando información que optimiza la relación con las demandas de la sociedad. La acreditación se orienta a fortalecer el papel de la autorregulación en la gestión de las instituciones, transparentando la diversidad de la oferta ante la sociedad y promoviendo mejores resultados en todo el sistema. Se trata de una práctica que sintetiza regulación e información sobre el mejoramiento de la calidad de la educación superior.

La propuesta de este ensayo es que la acreditación se perfila como el estímulo más relevante para la producción y difusión de información sobre la calidad de la educación superior, contribuyendo al mismo tiempo a la regulación del sistema. De este modo, la acreditación constituye una estrategia para enfrentar el problema de las asimetrías de la información de la educación terciaria.

Se discutirá sobre el problema de las asimetrías de información a partir de los intereses que predominan en los polos de coordinación del sistema de educación superior, para luego plantear cómo el proceso de acreditación contribuye a contrarrestar, en alguna medida, este problema a través de la información que se genera en sus distintas etapas, la cual permite caracterizar la calidad de esta educación. Finalmente, se plantean elementos para fortalecer la capacidad de los procesos de acreditación y producir y presentar información sobre la calidad de la educación.

\section{Asimetrías de información en la educación superior}

En este punto discutiremos acerca de las asimetrías de información que se producen en el sistema de educación superior cuando es uno de sus polos el que predomina sobre los otros, o bien cuando cada subsistema genera información de manera autorreferida. En estos 
casos, la información producida ofrece una imagen parcial de la calidad de la educación superior.

\subsection{El polo de las instituciones: publicidad y transparencia} de la educación superior

En sistemas de educación terciaria con significativa participación del mercado como mecanismo de coordinación, la publicidad se convierte en un medio fundamental de información. Según Squella (2001), en la educación superior chilena abunda la publicidad y falta información. La publicidad tiende a la homogenización de la información sobre la educación superior, en la medida en que obedece al interés transversal de las instituciones por favorecer su posicionamiento y prestigio ante los postulantes. Las instituciones tendrían más incentivos en asemejarse a un "tipo ideal" de institución en lugar de proporcionar a la sociedad una imagen comprehensiva de sus características.

El Departamento de Estudios de la CNA realizó este año una revisión de la información que las universidades chilenas proveían al público a través de sus páginas web. Para ello, se aplicó un checklist de información básica, considerando aspectos elementales como: (1) descripción de la institución, su misión, autoridades y sistema de toma de decisiones, (2) caracterización de la oferta académica, sus propósitos de formación, docentes y principales resultados, y (3) información sobre los servicios disponibles para los estudiantes. Como resultado, se verificó un $57 \%$ de cobertura promedio del checklist. Adicionalmente, se constató la existencia de diferencias importantes entre tipos de universidades: las tradicionales entregan más información pública que las universidades privadas nuevas, mientras que aquellas que están acreditadas también proporcionan más información que las que no lo están. Además, los años de acreditación de las universidades se asocian positivamente a los cinco mejores porcentajes de cobertura del checklist de información, promediando 4,8 años, efecto que se replica en los cinco menores porcentajes de cumplimiento del checklist, promediando 1,1 años de acreditación. En cuanto a la información relativa a calidad y desempeño, tales como los datos sobre eficiencia de la docencia, prácticamente no son informados por las universidades nacionales. Ello, a diferencia de lo 
que ocurre con las 30 primeras instituciones del ranking Shanghai Jiao Tong, que en un $70 \%$ cuentan con información de este tipo. Por lo tanto, el desempeño de las universidades en Chile ha sido bajo en cuanto a la información pública que proveen sobre la calidad de su oferta académica.

\subsection{El polo del mercado y la sociedad: necesidad de información y la función de los rankings}

Los rankings obedecen al interés social por diferenciar la oferta de educación superior. Ideados en 1983 por la revista US News and World Report, los rankings de educación superior son una de las herramientas más utilizadas actualmente para informarse sobre la calidad de las instituciones y sus programas. Ellos permiten ubicar a las instituciones en una tabla de posiciones, ordenada jerárquicamente según los puntajes que obtienen en una serie de indicadores que miden atributos como calidad y prestigio.

Los rankings representan una fuente de información limitada sobre calidad de la educación superior. Diversos autores (Usher y Savino, 2006; Dill y Soo, 2005; Marginson y Van Der Wende, 2006) sostienen que no son instrumentos de información fiables en la medida en que: las ponderaciones de los indicadores son subjetivas y arbitrarias; asumen que todos los usuarios le otorgan igual importancia a cada aspecto de la calidad; están basados en insumos y reputación más que en procesos y resultados; los resultados se entregan agregados institucionalmente y, en muchas ocasiones, son las mismas instituciones las que entregan los datos sin que haya mecanismos de validación. En el caso chileno, los rankings tienen un componente fundamental de "percepción de los usuarios"; representan entonces una "profecía autocumplida", ya que, en una buena medida, se construyen sobre base de opiniones representativas de los intereses que demandan y utilizan los rankings.

Los rankings presentan el riesgo de reducir la diversidad de la educación superior, al presentarla en un formato de gradaciones entre instituciones con misiones, orientaciones, funciones, insumos, procesos y resultados diferentes. 


\subsection{El polo del Estado: registros públicos}

Las estadísticas públicas representan un paso básico del polo regulatorio para influir en los procesos y resultados del sistema de educación superior. Tareas que van desde la asignación de recursos hasta los actuales esquemas de aseguramiento de la calidad se apoyan en información descriptiva sobre las instituciones, su oferta de programas, las características de los estudiantes que reciben y los desempeños en términos de eficacia de la gestión institucional, eficiencia del proceso de formación y equidad en los resultados.

Es junto con el crecimiento de la educación superior que la necesidad de contar con un mecanismo de información pública se vuelve imperativa. En esta década, el incremento en la cobertura de la educación postsecundaria ha alcanzado a casi el $40 \%$ de los jóvenes entre 18 y 24 años. Ello, de acuerdo con Trow (2007), ubica a Chile en un nivel masivo de su sistema de educación terciaria, caracterizado por una diversidad de estándares con los cuales medir los resultados y cruzado por nuevos desafíos, como la necesidad de implementar estrategias de nivelación para enfrentar los problemas de progresión y deserción en los estudiantes. En este contexto, la información se convierte en una exigencia fundamental para la política pública, los estudiantes y también para los empleadores. De otro modo es imposible conocer sobre los desempeños de las instituciones, establecer comparaciones con otros países y definir metas.

Sin embargo, la información descriptiva proporcionada por las estadísticas públicas tampoco permite, por sí misma, representar los niveles de calidad existentes en la educación superior. A través de datos como, por ejemplo, los puntajes de la Prueba de Selección Universitaria (PSU) nos podemos informar sobre la selectividad de una institución, así como sobre su orientación hacia la excelencia o hacia la inclusión social. Sin embargo, con los mismos datos no es posible conocer si dicha orientación institucional ha sido llevada a cabo asegurando la calidad.

Los principales sistemas de información son INDICES del Consejo Nacional de Educación y SIES del Ministerio de Educación (Mineduc), que cumplen funciones similares: generar estadísticas 
descriptivas en el ámbito nacional. SIES, en particular, ha implementado la utilización del Rol Único Tributario de estudiantes y académicos, lo que permite acceder a información más completa que la que existía antes. Con ello se abre la posibilidad de hacer un seguimiento a cada cohorte y conocer los desempeños en cuanto a la progresión de los estudiantes y eficiencia del proceso de formación, así como también conocer acerca de la empleabilidad de los egresados. Estos son avances sustantivos que permiten transformar información descriptiva de las estadísticas oficiales en información compleja sobre la calidad de la educación superior.

Pese a que la existencia de estos dos sistemas para un mismo propósito no representa un problema, sí lo es el que no haya suficiente integración entre ellos, al mismo tiempo que hay disparidad en la información entregada y, además, el que una parte importante de la realidad del sistema de educación superior chilena, compuesta por programas y sedes no declaradas, queda fuera de las estadísticas oficiales.

Cabe preguntarse si la generación de información sobre la calidad de la educación superior, que permite distinguir los diferentes tipos de instituciones y programas, resulta de la maximización de la información producida, de manera particular, por las instituciones, la sociedad/mercado y el Estado. Para que un sistema de educación terciaria pueda generar información sobre su calidad y superar las asimetrías de información, es necesario alinear el rol regulador del Estado con la capacidad de autorregulación de las instituciones, de manera de generar indicadores y parámetros que den cuenta de los desempeños del sistema a la vez que transparenten la heterogeneidad del mercado ante los usuarios. Sostenemos que esta información va necesariamente asociada a la regulación. No se trata de términos reemplazables. Asimismo, la regulación no se constituye como una función exclusiva del Estado, sino que la calidad se produce cuando la regulación es asumida como un ejercicio público de rendición de cuentas por parte de las instituciones. La acreditación representa el mecanismo e incentivo que produce esta articulación entre regulación e información sobre calidad de la educación superior. 


\section{La acreditación como fuente de información}

En Chile la acreditación ha sido la principal fuente de información sobre calidad de la educación superior. En los puntos siguientes se explica la forma en que cumple este rol estratégico para el sistema.

\subsection{Niveles de información}

Es necesario distinguir dos niveles de información. El primero corresponde a la información básica sobre el sistema de educación superior; el segundo apunta a información sobre su calidad, como se observa en la figura 1. El nivel de la información básica se compone de datos como tipo y número de instituciones, oferta de carreras, matrícula, número de profesores e infraestructura. Los sistemas de información existentes (SIES e INDICES) corresponden a este nivel de información.

Figura 1: Niveles de información

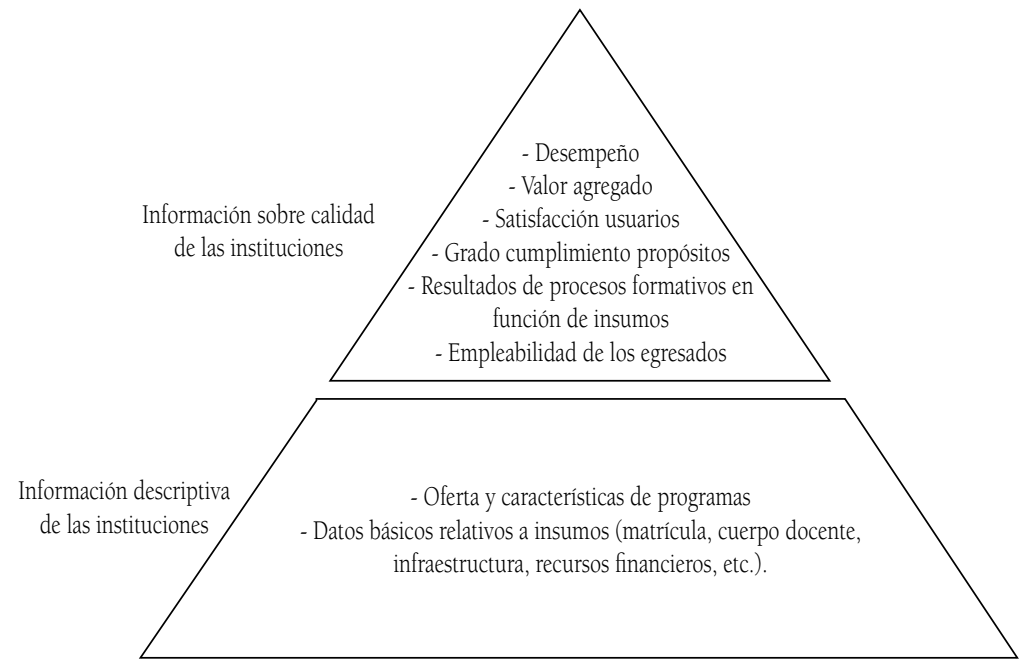

Fuente: elaboración propia

El segundo nivel de información sobre la calidad de la educación superior se ha desarrollado en Chile a partir de los procesos de acreditación, consistente en información que certifica los niveles de calidad existentes en la educación superior. Este proceso incentiva 
acciones de mejoramiento de parte de las instituciones y sus carreras. En este sentido, promueve un uso estratégico de la información, en el sentido de ajustar las políticas, mecanismos y acciones realizadas por las instituciones y carreras hacia mejores resultados (Middaugh, 2009). La acreditación promueve la gestión de la información como estrategia de autorregulación.

La acreditación sintetiza un enfoque cuantitativo, como tasas de graduados o libros en la biblioteca, con un análisis cualitativo de los procesos y sistemas para el mejoramiento institucional (Young, Chambers y Kells, 1983). Por lo tanto, la capacidad de un sistema de educación superior de generar información sobre su calidad depende directamente de la capacidad de las instituciones y de las agencias públicas para implementar estrategias de regulación que utilicen la información del primer nivel para convertirla en metas de mejoramiento.

\subsection{Etapas de la acreditación}

Para una institución de educación superior cada una de las etapas del proceso de acreditación permite generar información del segundo nivel, relevante para la gestión interna y para la regulación del sistema, en la medida en que favorece la coordinación con los polos de la sociedad/mercado y autoridad estatal.

En primer lugar, el proceso de autoevaluación, en el que por ley participan los distintos estamentos de las comunidades institucionales, produce información acerca de la misión, visión y valores particulares de cada institución, así como de sus entornos internos y externos, representando una oportunidad de difusión interna y de socialización de la "cultura institucional". Además, la autoevaluación genera datos nuevos, actualiza información antigua y, lo más importante, representa una instancia de consulta a informantes claves de las instituciones (estudiantes, profesores, directivos) y stakeholders: empleadores y figuras relevantes del medio.

En segundo lugar, la evaluación de los pares contribuye con una mirada externa que se refiere la efectividad de las acciones emprendidas por las instituciones para responder a las debilidades identificadas y para 
proyectar su misión en el tiempo. Para las instituciones, la evaluación externa podría asemejarse a una auditoría que valida el análisis llevado a cabo en la evaluación interna, a la vez que contrasta sus resultados con la perspectiva multidisciplinaria del Comité de Pares. Para la Comisión, la evaluación externa constituye una instancia de interpretación de información que traduce y organiza significativamente datos del primer nivel en información del segundo nivel.

Por último, el pronunciamiento de la Comisión está fundamentado en argumentos presentados como "acuerdos de acreditación". Estos acuerdos resumen el nivel de cumplimiento de la institución de los estándares de calidad definidos en los términos de referencia; se pronuncian sobre sus resultados en áreas relevantes como la efectividad de la docencia, la investigación y la viabilidad financiera, y se refieren al desarrollo de las instituciones a partir de la solidez de los sistemas de autorregulación que han implementado. Los acuerdos de acreditación son públicos y constituyen información relevante para todos los usuarios ${ }^{2}$. La Ley que crea la CNA establece como una de sus funciones proveer información a los distintos usuarios a través de la difusión de los acuerdos de acreditación.

Los resultados de acreditación son la principal fuente pública de información sobre calidad de la educación superior en Chile. En Estados Unidos la información emanada de estos procesos constituye un elemento fundamental para la toma de decisiones. De acuerdo con los resultados de una encuesta aplicada en 1999 por el Consejo para la Acreditación de la Educación Superior (CHEA, en inglés), un 55\% cree que las universidades deben cumplir con altos estándares para recibir la acreditación. Por otro lado, un 66\% manifestó que no consideraría ingresar a una carrera de una institución que no está acreditada.

\subsection{Reflexividad de la educación superior}

La idea de "reflexividad" pertenece a Giddens (1990). Aplicada al contexto de una institución de educación superior, se puede definir

2 La página web http://www.cnachile.cl recibió durante el mes de enero de 2009, periodo de postulaciones, un promedio de 2.316 visitas diarias, en contraste con un mes normal que tiene alrededor de 600 visitas diarias. 
como la revisión constante de las prácticas, examinadas y reformuladas a la luz de información emanada a partir de esas mismas prácticas, de tal modo que constitutivamente alteran su carácter, generando un sistema activo de aprendizaje institucional. Sostenemos que la acreditación constituye un uso reflexivo de la información. Primero, porque ha conducido a que instituciones y carreras desarrollen sistemas de información para controlar los procesos internos, orientar estratégicamente la gestión hacia objetivos y mejorar el posicionamiento en el medio externo mediante diagnósticos informados, que consideran el desempeño de estudiantes y egresados. Segundo, porque determina un incremento en la "reflexividad" del sistema de educación superior, en la medida en que produce información que motiva el mejoramiento del conjunto, al mismo tiempo que promueve la diferenciación, es decir, que las instituciones vayan especificando sus ámbitos de desarrollo. Sobre esto último, Trow (1996) sostiene que los procesos internos de aseguramiento de la calidad son los que llevan al mejoramiento de la calidad, en oposición a los procesos externos, que tienen un sesgo hacia la accountability.

Uno de los efectos claros de la acreditación ha sido la implementación de sistemas de información que apoyan a las políticas y mecanismos de autorregulación. Si una institución o carrera está acreditada significa que, en alguna medida, ha desarrollado información orientada al propósito de asegurar la calidad. Desde la puesta en práctica de los procesos experimentales de acreditación, la mayoría de las instituciones cuenta con oficinas que trabajan permanentemente en la conducción de procesos de evaluación interna. Por ejemplo, un área donde antes de la acreditación existía un desarrollo prácticamente nulo es en el seguimiento de los egresados. Actualmente, instituciones y carreras, en mayor o menor medida, han institucionalizado procesos de seguimiento de resultados, mediante la información proporcionada por el desempeño de los egresados, que se utiliza para retroalimentar los procesos académicos.

La acreditación ha promovido este nivel de ajuste interno en las instituciones de educación superior, pero sin necesariamente sacrificar la diversidad del sistema. La discusión que acompañó la definición de la Ley 20.129 estuvo orientada sobre todo al riesgo de homogeneizar 
el sistema de educación superior a través de la acción centralizada de una agencia nacional de acreditación. Como alternativa, se planteaba la necesidad de generar un sistema de información que señalara los diferentes niveles de calidad. Pero, ¿cómo se construye un sistema de información de la calidad sin que se lleven a cabo procesos de acreditación? Sin aseguramiento de la calidad, la información que se pueda construir sobre el sistema sólo puede ser del primer nivel: información descriptiva, general y comparable, pero no información que señale la diversidad de misiones institucionales, de perfiles de egreso y de resultados de políticas y mecanismos de aseguramiento de la calidad. La acreditación es en sí un sistema de información sobre la calidad que no necesariamente homogeniza la oferta académica y las orientaciones de las instituciones, sino que parte del reconocimiento de cada misión institucional y de los perfiles de egreso, y sobre estas definiciones básicas construye la evaluación ${ }^{3}$. Como plantean Letelier y Carrasco (2004), la acreditación es un análisis de las relaciones causales entre los propósitos establecidos por la institución o carrera y sus actividades, recursos, resultados e impacto social.

\section{Información sobre calidad de la educación superior}

La acreditación en Chile es un sistema relativamente nuevo. Existen problemas que se deben superar para potenciar la capacidad de la acreditación de generar información sobre la calidad de la educación superior. Estudios concluyen que la implementación de los sistemas de aseguramiento de la calidad en la educación terciaria no es un simple proceso lineal, sino más bien uno altamente complejo y, en algunas ocasiones, un proceso paradójico y contradictorio (Stensaker, 2007). A continuación se explican algunos de estos problemas y se plantean líneas de desarrollo.

La acreditación también reproduce asimetrías en la generación de información sobre la calidad de la educación superior. En primer

3 Sin embargo, a pesar del concepto bien aceptado de que la calidad y su evaluación se mide referida a los propósitos de las instituciones, enfatizando las dimensiones subjetivas y la multidimensionalidad del concepto, existe aún necesidad de enfoques más avanzados para desarrollar el concepto de calidad en la educación superior (Stensaker, 2007). 
lugar, existe una tensión entre la necesidad de proveer información a los usuarios del sistema, como un acto de dar "fe pública", con la necesaria prudencia y confidencialidad que acompaña el desarrollo de las etapas del proceso de acreditación (Ewell, 2004). Es decir, la acreditación no puede ser "no selectiva" en la información que proporciona a los usuarios, pues ello puede alterar el desarrollo de las etapas del proceso.

En segundo lugar, los resultados de los procesos de acreditación producen información parcial sobre la calidad en dos sentidos. Porque representa un momento concreto: una institución puede empeorar o mejorar luego del dictamen de acreditación. Y porque entrega información sobre una parte y no la totalidad del quehacer institucional. Por lo tanto, la acreditación podría generar asimetrías de información en virtud de la evidencia empírica "que sugiere la existencia de grandes diferencias de desempeño entre diferentes áreas en una misma universidad. Una universidad puede tener un buen ranking en física al mismo tiempo que ser rankeada muy bajo en literatura" (Federkeil, 2002). Los acuerdos de acreditación producidos como resultado de todo el proceso reflejan la realidad general de una institución o carrera, lo cual podría representar una limitación ante usuarios que requieren información más específica para tomar sus decisiones.

En tercer término, quienes participan en el proceso de acreditación, en la medida en que esperan obtener un resultado positivo, podrían presentar información de manera "estratégica", es decir, relevando algunos antecedentes sobre el resto e, incluso, ocultando información que puede ser clave para la evaluación. La acreditación es un proceso de carácter voluntario que parte de la "buena fe". Sin embargo, el valor que adquiere el sello de la acreditación ante la preferencia de los postulantes, junto con el carácter obligatorio que ha adquirido la acreditación de carreras de pedagogía y medicina, introducen incentivos para adecuar la información con el fin de obtener un buen resultado de acreditación. Frente a este efecto, la evaluación de los pares actúa como instancia de validación de la información presentada en la autoevaluación. Incluso, en áreas específicas del funcionamiento de las instituciones, la evaluación externa se perfila como una auditoría. Con todo, 
esto no implica que la acreditación garantice que la información entregada en los informes sea absolutamente fidedigna, pero, de alguna manera, a través de las distintas etapas de la acreditación se puede obtener información más completa y confiable sobre la calidad de las instituciones y programas académicos. Un ejemplo interesante en este punto es la información sobre matrícula y sedes. Según el informe de la OECD (2009), las instituciones tienen interés en mostrar una mayor proporción de matrículas en su casa central o sede principal más que en sus otros recintos universitarios. De este modo, los registros públicos del Ministerio de Educación (Mineduc) y del CSE en ocasiones han mostrado un número inferior a las sedes y matrícula que en realidad tiene una institución ${ }^{4}$. Esta información ha sido transparentada, en una buena medida, a partir de los procesos de acreditación, ya que la verificación en terreno de los datos presentados en la autoevaluación implica una presión por mayor transparencia en la información proveída para la evaluación.

La acreditación requiere fortalecer algunos aspectos con el fin de robustecer su capacidad de proveer información sobre la calidad de la educación superior. Por ejemplo, si bien la información que entregan las instituciones y programas es generada en el marco procesos cuidadosos y reflexivos, que contemplan la consulta a informantes clave y que luego es verificada en la etapa de evaluación externa, no hay mecanismos formales y establecidos de auditoría que permitan cautelar que la información sea verdadera y completa. Otro ejemplo es que hoy los distintos procesos de acreditación -institucional, de carreras y posgrados-son independientes, de modo que una institución puede estar acreditada por docencia de pregrado sin tener ninguna carrera acreditada. Ello representa una limitación desde el punto de vista de la información porque, como se planteó, la acreditación institucional no arroja necesariamente información detallada sobre la calidad de programas específicos de la oferta académica. Para resolver este problema no se trata de condicionar mutuamente los resultados obtenidos en los diferentes tipos de acreditación, sino más bien de asociar la información que arrojan los resultados de acreditación,

4 Un estudio realizado por la CNA contó 141 sedes de instituciones de educación superior en el país que no figuraban en los registros oficiales. 
de tal forma de ofrecer una imagen comprehensiva sobre la calidad de la oferta académica. La experiencia internacional de sistemas de información, tales como el UNISTATS de Reino Unido y el ranking del Centre for Higher Education Development (CHE) de Alemania, es clara en este sentido, dando cuenta del interés creciente de los estudiantes por acceder a información sobre la calidad de los programas impartidos por instituciones de educación terciaria.

Una línea de desarrollo para fortalecer la capacidad de la acreditación de generar información sobre la calidad de la educación superior consiste en organizar y presentar antecedentes cualitativos y cuantitativos complementarios a los juicios de acreditación. Esto significa ir más allá en la función establecida por Ley de difundir los resultados del proceso de acreditación. Se trata de avanzar en la construcción de un sistema de información sobre la calidad de la educación superior que dé cuenta de los resultados obtenidos por instituciones, carreras y programas en la aplicación de las políticas y mecanismos de aseguramiento de la calidad; implica asociar la presentación la información de los resultados de acreditación contenida en los respectivos acuerdos con indicadores relevantes sobre áreas críticas, como dotación académica, efectividad de la docencia, capacidad de autorregulación o productividad científica. Este tipo de indicadores, en particular aquellos sobre efectividad de la docencia, han sido especialmente considerados para caracterizar la calidad de la educación impartida por una institución (Dill y Soo, 2004; Trow, 1996). La probabilidad que tiene un estudiante, que cumple con las condiciones de admisión de una institución, de mantenerse cursando un programa y terminarlo a tiempo es un dato relevante desde el punto de vista de la calidad de la educación superior. Con todo, la orientación no es a objetivar los juicios de acreditación en indicadores cuantitativos concretos como única fuente de información, sino que a integrarlos a la caracterización cualitativa sobre la efectividad de las políticas y mecanismos de aseguramiento de la calidad definidos e implementados por las instituciones que participan en procesos de acreditación ${ }^{5}$.

5 Es interesante considerar la sugerencia del Higher Education Funding Council for England a las universidades en cuanto a que éstas deben informar en sus páginas web sobre sus políticas de aseguramiento de la calidad y los resultados obtenidos de su aplicación.

Véase http://www.hefce.ac.uk/Pubs/hefce/2002/02_15/02_15.doc 
Proporcionar información sobre la calidad de la educación superior, complementaria a los resultados de acreditación, representaría un aporte a la transparencia del sistema. Por una parte, la información sobre la calidad permite dar a conocer la diversidad del sistema de educación superior: las instituciones reciben diferentes estudiantes, con distintas necesidades e implementan diferentes modelos de formación orientados a atenderlas, los cuales, a su vez, requieren de diferentes recursos organizacionales, humanos y de infraestructura, y diferentes estrategias de aseguramiento de la calidad; finalmente, presentan una diversidad de resultados y maneras de expresarlos. Por otro lado, la información es fundamental para apoyar la toma de decisiones de los usuarios. Por ejemplo, el estudio sobre las causas de la deserción universitaria, conducido por el Centro de Microdatos de la Universidad de Chile (2008), muestra que junto con que los estudiantes demandan el aumento de información sobre las carreras, un alto porcentaje de directivos de las universidades señalan que la desinformación de los estudiantes constituye una de las principales causas de las elevadas tasas de deserción.

El papel de la acreditación se vería sustantivamente reforzado si se combina con un sistema de información ad hoc. De este modo, se abriría aún más el acceso a información sobre la calidad de la educación superior a los usuarios, reduciendo las asimetrías existentes en este ámbito. Al mismo tiempo, las instituciones de educación superior contarían con parámetros significativos para orientar sus acciones estratégicas y de mejoramiento (benchmarking). Por último, la implementación de un sistema de información sobre la calidad implicará un paso más en la consolidación de la acreditación en Chile, en el sentido de incrementar su capacidad para evaluar resultados, propender al mejoramiento de estándares y monitorear las variables críticas que influyen sobre la calidad de la educación.

\section{Conclusiones}

La proposición de este ensayo es que la acreditación se constituye como el estímulo más relevante para la producción y difusión de información sobre la calidad de la educación superior, contribuyendo a la regulación de todo el sistema. De esta manera, en lugar de tratarse 
de estrategias excluyentes o sustituibles para mejorar la calidad de la educación superior en Chile, se trata de un círculo virtuoso, en el cual regulación e información se refuerzan mutuamente.

El sistema de educación superior chileno produce información siguiendo los intereses de sus distintos polos de funcionamiento: las instituciones, el Estado y la sociedad/mercado. De acuerdo con nuestra caracterización, las instituciones buscan mostrar las cualidades de su oferta a los postulantes utilizando, en una buena medida, la publicidad para ello. El Estado genera información con el fin de llevar un registro sobre las dinámicas de crecimiento y diferenciación del sistema, pero este proceso no se ha llevado a cabo sin problemas de asimetrías de información, a la vez que no se ha logrado articular, de manera sistemática, información descriptiva con aquella que da cuenta de los niveles de calidad del sistema. La sociedad y el mercado han recurrido a la generación de rankings para informar de las posiciones y el prestigio de las instituciones. Ninguno de estos tres intereses, por sí sólo, ha logrado producir de manera consistente información que reporte la calidad y diversidad de la educación superior chilena. Para que el sistema cuente con información acerca de su calidad, que le permita mejorar sus desempeños globales, es necesario fortalecer la capacidad de coordinación entre los diferentes subsistemas. Este es el papel de la acreditación: generar información que asocie los resultados de los esfuerzos de autorregulación de las instituciones con los estándares definidos desde el polo de regulación del sistema, con el fin de promover su mejoramiento.

El desarrollo del aseguramiento de la calidad informa de pasos decididos hacia la generación de información sobre calidad de la educación superior que, aunque no exenta de problemas y asimetrías, sirve a instituciones, estudiantes, usuarios y a los fines de la regulación pública. La información sobre calidad constituye una herramienta fundamental para la toma de decisiones, de forma que el desarrollo de un sistema de información, basado en los procesos de acreditación, representa una oportunidad para contribuir con la transparencia de todo el sistema y dar cuenta de la diversidad de misiones, recursos, procesos y resultados que caracterizan a la educación superior chilena. 
En la medida en que las instituciones sigan haciendo del aseguramiento de la calidad una práctica constante y vinculada a las decisiones estratégicas, el resultado lógico será un incremento en la calidad de la información disponible, tanto para la gestión interna como para el medio externo. Asimismo, en la medida en que la acreditación se complemente con más y más diversa información, los ejercicios de autoevaluación, los informes de los pares evaluadores y los juicios de acreditación serán representaciones cada vez más fieles de los esfuerzos colectivos para el mejoramiento de la educación postsecundaria.

\section{Referencias bibliográficas}

Brunner, José Joaquín (2004) Políticas y mercado de educación superior: necesidades de información. En Brunner, José Joaquín y Meller, Patricio (comps.) Oferta y demanda de profesionales y técnicos en Chile. El rol de la información pública. Santiago de Chile: Ril Editores.

Brunner, José Joaquín (2008) Educación superior en Chile: instituciones, Mercado y politicas gubernamentales, 1967-2007. Holanda: Universidad de Leiden.

Centro de Microdatos (2008) Estudio Sobre Causas de la Deserción Universitaria. Departamento de Economía. Santiago de Chile: Universidad de Chile.

CHEA: Letter from the President. Sitio en Internet. Disponible en http://www. chea.org/Research/president-letters/99-07-16.asp

CINDA (2006) Repitencia y Deserción Universitaria en América Latina. Santiago de Chile: CINDA.

Clark, Burton (1983) The Higher Education System. Berkeley: University of California Press.

CSE (2008) Retención en el sistema de educación superior: seguimiento de los cohortes 2004, 2005 y 2006. Santiago de Chile: CSE.

Dill, David y Soo, Maarja (2004) Transparency and quality in Higher Education markets. En Teixeira et al. (eds.) Markets in Higher Education: Rhetoric of reality? Netherlands: Higher Education Dynamics, Douro Series.

Dill, David y Soo, Maarja (2005) Academic quality, league tables, and public policy: A cross-national analysis of university ranking systems. Higher Education, 49, pp. 495-533. 
Ewell, Peter (2004) Accreditation and the provision of additional information to the public about institution and public performance. Washington D.C.: CHEA Occasional Paper.

Giddens, Anthony (1990) The consequences of modernity. Stanford, California: Stanford University Press.

HEFCE (2002) Information on quality and standards in higher education. Final Report of the Task Group, Reino Unido: Higher Education Funding Council for England.

Letelier, Mario y Carrasco, Rosario (2004) Higher Education assessment and accreditation in Chile: state-of-the-art and trends. European Journal of Engineering Education, 29(1), pp. 119-124.

Marginson, Simon and Van Der Wende, Marijk (2007) To Rank Or To Be Ranked: The Impact of Global Rankings in Higher Education. Journal of Studies in International Education, 11(3-4), pp. 306-329.

Middaugh, Michael (2009) Closing the loop: liking planning and assessment. Planning for Higher Education, 37(3), pp. 5-14.

OECD (2009) La educación superior en Chile: Revisión de Políticas Nacionales de Educación. OECD/BIRD/Banco Mundial.

Squella, Agustín (2001) Información en el marco de la calidad de la educación superior. CSE/CNAP: Información en la educación superior. Necesidades y propuestas. Santiago de Chile: CSE.

Stensaker, Bjorn (2007) Quality as fashion: exploring the translation of management idea into higher education. Quality Assurance in Higher Education: Trends in regulation, translation and transformation. Springer, pp. 99-118.

Trow, Martin (1996) Trust, markets, and accountability in Higher Education: a comparative perspective. Higher Education Policy, 9(4).

Trow, Martin (2007) Reflections on the transition from elite to mass to universal access: forms and phases of higher education in modern societies since WWII. In Forest, James J. F. and Altbach, Philip G. (eds.) International Handbook of Higher Education. Springer.

Usher, Alex y Savino, Massimo (2006) A world of difference: A Global Survey of University League Tables. Canadian Education Report Series.

Young, Kenneth; Chambers, Charles y Kells, H.R. (1983) Understanding accreditation. San Francisco: Jossey-Bass.

Recibido: 18 de junio de 2009

Aceptado: 15 de octubre de 2009 